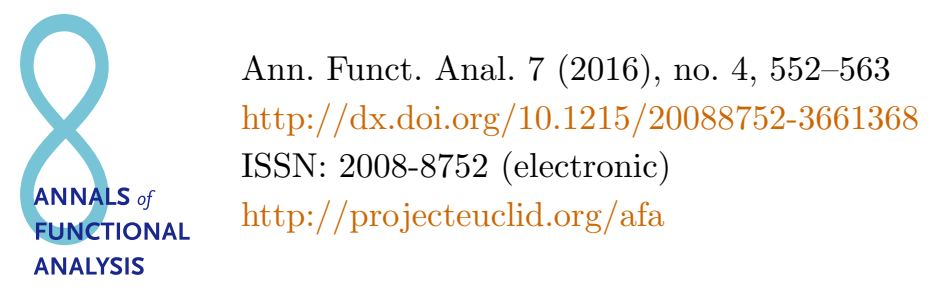

\title{
PRODUCTS OF LAURENT OPERATORS AND FIELDS OF VALUES
}

\author{
NATÁLIA BEBIANO $^{1^{*}}$ and JOÃO DA PROVIDÊNCIA ${ }^{2}$ \\ Communicated by I. M. Spitkovsky
}

\begin{abstract}
One of the most fundamental properties of the field of values of an operator is the inclusion of the spectrum within its closure. Obtaining information on the spectrum of products of operators in terms of this spectral inclusion region is a demanding issue. Stating general results seems difficult; however, conclusions can be derived in some special instances. In this paper, we show that the field of values of products of Laurent operators is easily related to the product of their fields of values, and the same occurs for certain classes of Laurent operators with matrix symbols. The results also apply to the class of infinite upper (lower) triangular Toeplitz matrices.
\end{abstract}

\section{INTRODUCTION}

Let $A$ be a bounded operator on a Hilbert space $H$ equipped with an inner product $\langle$,$\rangle . Denote by B(H)$ the algebra of bounded linear operators over $H$. In our discussion, we identify $H$ with $\mathbb{C}^{n}$ whenever $H$ has dimension $n$. The field of values of $A$ is the set of the complex plane defined as

$$
W(A)=\{\langle A f, f\rangle /\langle f, f\rangle: f \in H,\langle f, f\rangle \neq 0\} .
$$

This concept is a useful tool in studying linear operators, and it has been extensively investigated (see, e.g., [4] and the references therein).

Copyright 2016 by the Tusi Mathematical Research Group.

Received Mar. 4, 2016; Accepted Mar. 20, 2016.

${ }^{*}$ Corresponding author.

2010 Mathematics Subject Classification. Primary 46C20; Secondary 47A12, 47A10, 47B35.

Keywords. fields of values, spectrum, Toeplitz operator, Laurent operator, symbol. 


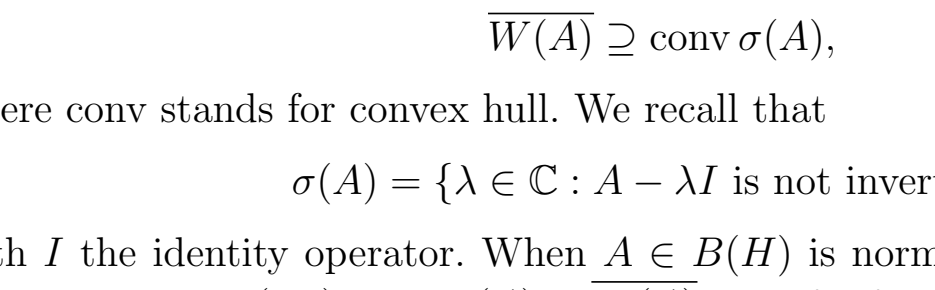

\title{
PRODUCTS OF LAURENT OPERATORS AND FIELDS OF VALUES
}

\author{
NATÁLIA BEBIANO $^{1^{*}}$ and JOÃO DA PROVIDÊNCIA ${ }^{2}$ \\ Communicated by I. M. Spitkovsky
}

\begin{abstract}
One of the most fundamental properties of the field of values of an operator is the inclusion of the spectrum within its closure. Obtaining information on the spectrum of products of operators in terms of this spectral inclusion region is a demanding issue. Stating general results seems difficult; however, conclusions can be derived in some special instances. In this paper, we show that the field of values of products of Laurent operators is easily related to the product of their fields of values, and the same occurs for certain classes of Laurent operators with matrix symbols. The results also apply to the class of infinite upper (lower) triangular Toeplitz matrices.
\end{abstract}

\section{INTRODUCTION}

Let $A$ be a bounded operator on a Hilbert space $H$ equipped with an inner product $\langle$,$\rangle . Denote by B(H)$ the algebra of bounded linear operators over $H$. In our discussion, we identify $H$ with $\mathbb{C}^{n}$ whenever $H$ has dimension $n$. The field of values of $A$ is the set of the complex plane defined as

$$
W(A)=\{\langle A f, f\rangle /\langle f, f\rangle: f \in H,\langle f, f\rangle \neq 0\} .
$$

This concept is a useful tool in studying linear operators, and it has been extensively investigated (see, e.g., [4] and the references therein).

Copyright 2016 by the Tusi Mathematical Research Group.

Received Mar. 4, 2016; Accepted Mar. 20, 2016.

${ }^{*}$ Corresponding author.

2010 Mathematics Subject Classification. Primary 46C20; Secondary 47A12, 47A10, 47B35.

Keywords. fields of values, spectrum, Toeplitz operator, Laurent operator, symbol. 


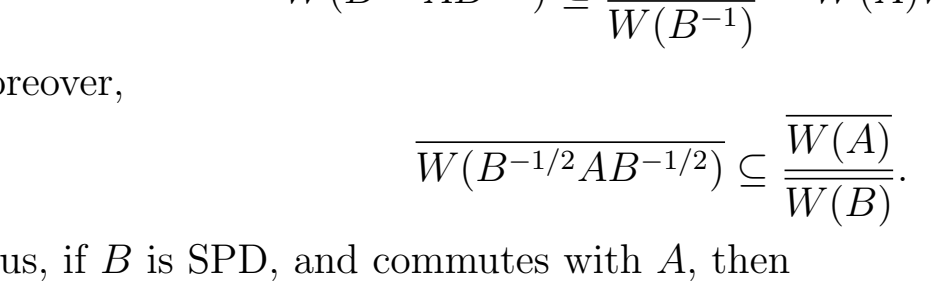

\title{
PRODUCTS OF LAURENT OPERATORS AND FIELDS OF VALUES
}

\author{
NATÁLIA BEBIANO $^{1^{*}}$ and JOÃO DA PROVIDÊNCIA ${ }^{2}$ \\ Communicated by I. M. Spitkovsky
}

\begin{abstract}
One of the most fundamental properties of the field of values of an operator is the inclusion of the spectrum within its closure. Obtaining information on the spectrum of products of operators in terms of this spectral inclusion region is a demanding issue. Stating general results seems difficult; however, conclusions can be derived in some special instances. In this paper, we show that the field of values of products of Laurent operators is easily related to the product of their fields of values, and the same occurs for certain classes of Laurent operators with matrix symbols. The results also apply to the class of infinite upper (lower) triangular Toeplitz matrices.
\end{abstract}

\section{INTRODUCTION}

Let $A$ be a bounded operator on a Hilbert space $H$ equipped with an inner product $\langle$,$\rangle . Denote by B(H)$ the algebra of bounded linear operators over $H$. In our discussion, we identify $H$ with $\mathbb{C}^{n}$ whenever $H$ has dimension $n$. The field of values of $A$ is the set of the complex plane defined as

$$
W(A)=\{\langle A f, f\rangle /\langle f, f\rangle: f \in H,\langle f, f\rangle \neq 0\} .
$$

This concept is a useful tool in studying linear operators, and it has been extensively investigated (see, e.g., [4] and the references therein).

Copyright 2016 by the Tusi Mathematical Research Group.

Received Mar. 4, 2016; Accepted Mar. 20, 2016.

${ }^{*}$ Corresponding author.

2010 Mathematics Subject Classification. Primary 46C20; Secondary 47A12, 47A10, 47B35.

Keywords. fields of values, spectrum, Toeplitz operator, Laurent operator, symbol. 


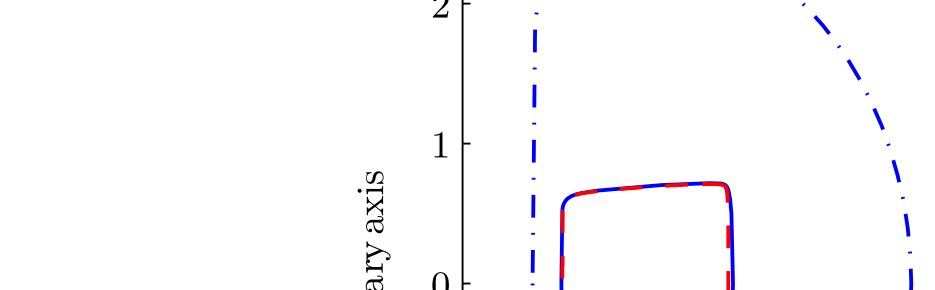

\title{
PRODUCTS OF LAURENT OPERATORS AND FIELDS OF VALUES
}

\author{
NATÁLIA BEBIANO $^{1^{*}}$ and JOÃO DA PROVIDÊNCIA ${ }^{2}$ \\ Communicated by I. M. Spitkovsky
}

\begin{abstract}
One of the most fundamental properties of the field of values of an operator is the inclusion of the spectrum within its closure. Obtaining information on the spectrum of products of operators in terms of this spectral inclusion region is a demanding issue. Stating general results seems difficult; however, conclusions can be derived in some special instances. In this paper, we show that the field of values of products of Laurent operators is easily related to the product of their fields of values, and the same occurs for certain classes of Laurent operators with matrix symbols. The results also apply to the class of infinite upper (lower) triangular Toeplitz matrices.
\end{abstract}

\section{INTRODUCTION}

Let $A$ be a bounded operator on a Hilbert space $H$ equipped with an inner product $\langle$,$\rangle . Denote by B(H)$ the algebra of bounded linear operators over $H$. In our discussion, we identify $H$ with $\mathbb{C}^{n}$ whenever $H$ has dimension $n$. The field of values of $A$ is the set of the complex plane defined as

$$
W(A)=\{\langle A f, f\rangle /\langle f, f\rangle: f \in H,\langle f, f\rangle \neq 0\} .
$$

This concept is a useful tool in studying linear operators, and it has been extensively investigated (see, e.g., [4] and the references therein).

Copyright 2016 by the Tusi Mathematical Research Group.

Received Mar. 4, 2016; Accepted Mar. 20, 2016.

${ }^{*}$ Corresponding author.

2010 Mathematics Subject Classification. Primary 46C20; Secondary 47A12, 47A10, 47B35.

Keywords. fields of values, spectrum, Toeplitz operator, Laurent operator, symbol. 


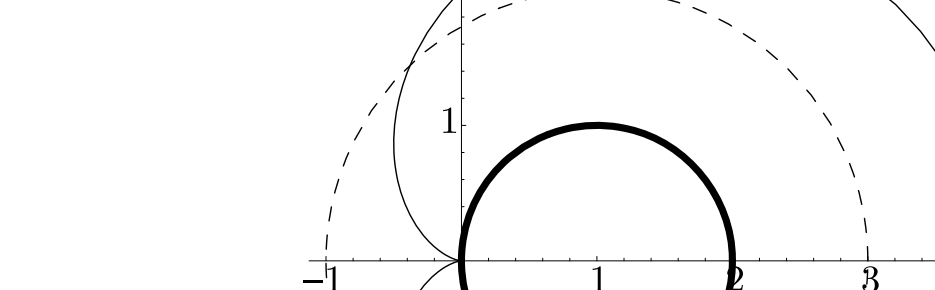

\title{
PRODUCTS OF LAURENT OPERATORS AND FIELDS OF VALUES
}

\author{
NATÁLIA BEBIANO $^{1^{*}}$ and JOÃO DA PROVIDÊNCIA ${ }^{2}$ \\ Communicated by I. M. Spitkovsky
}

\begin{abstract}
One of the most fundamental properties of the field of values of an operator is the inclusion of the spectrum within its closure. Obtaining information on the spectrum of products of operators in terms of this spectral inclusion region is a demanding issue. Stating general results seems difficult; however, conclusions can be derived in some special instances. In this paper, we show that the field of values of products of Laurent operators is easily related to the product of their fields of values, and the same occurs for certain classes of Laurent operators with matrix symbols. The results also apply to the class of infinite upper (lower) triangular Toeplitz matrices.
\end{abstract}

\section{INTRODUCTION}

Let $A$ be a bounded operator on a Hilbert space $H$ equipped with an inner product $\langle$,$\rangle . Denote by B(H)$ the algebra of bounded linear operators over $H$. In our discussion, we identify $H$ with $\mathbb{C}^{n}$ whenever $H$ has dimension $n$. The field of values of $A$ is the set of the complex plane defined as

$$
W(A)=\{\langle A f, f\rangle /\langle f, f\rangle: f \in H,\langle f, f\rangle \neq 0\} .
$$

This concept is a useful tool in studying linear operators, and it has been extensively investigated (see, e.g., [4] and the references therein).

Copyright 2016 by the Tusi Mathematical Research Group.

Received Mar. 4, 2016; Accepted Mar. 20, 2016.

${ }^{*}$ Corresponding author.

2010 Mathematics Subject Classification. Primary 46C20; Secondary 47A12, 47A10, 47B35.

Keywords. fields of values, spectrum, Toeplitz operator, Laurent operator, symbol. 


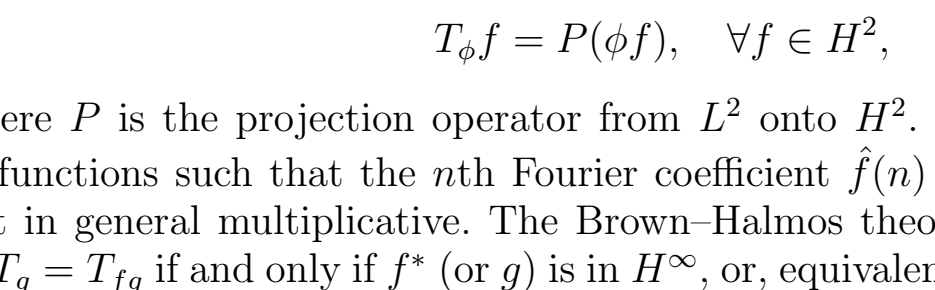

\title{
PRODUCTS OF LAURENT OPERATORS AND FIELDS OF VALUES
}

\author{
NATÁLIA BEBIANO $^{1^{*}}$ and JOÃO DA PROVIDÊNCIA ${ }^{2}$ \\ Communicated by I. M. Spitkovsky
}

\begin{abstract}
One of the most fundamental properties of the field of values of an operator is the inclusion of the spectrum within its closure. Obtaining information on the spectrum of products of operators in terms of this spectral inclusion region is a demanding issue. Stating general results seems difficult; however, conclusions can be derived in some special instances. In this paper, we show that the field of values of products of Laurent operators is easily related to the product of their fields of values, and the same occurs for certain classes of Laurent operators with matrix symbols. The results also apply to the class of infinite upper (lower) triangular Toeplitz matrices.
\end{abstract}

\section{INTRODUCTION}

Let $A$ be a bounded operator on a Hilbert space $H$ equipped with an inner product $\langle$,$\rangle . Denote by B(H)$ the algebra of bounded linear operators over $H$. In our discussion, we identify $H$ with $\mathbb{C}^{n}$ whenever $H$ has dimension $n$. The field of values of $A$ is the set of the complex plane defined as

$$
W(A)=\{\langle A f, f\rangle /\langle f, f\rangle: f \in H,\langle f, f\rangle \neq 0\} .
$$

This concept is a useful tool in studying linear operators, and it has been extensively investigated (see, e.g., [4] and the references therein).

Copyright 2016 by the Tusi Mathematical Research Group.

Received Mar. 4, 2016; Accepted Mar. 20, 2016.

${ }^{*}$ Corresponding author.

2010 Mathematics Subject Classification. Primary 46C20; Secondary 47A12, 47A10, 47B35.

Keywords. fields of values, spectrum, Toeplitz operator, Laurent operator, symbol. 


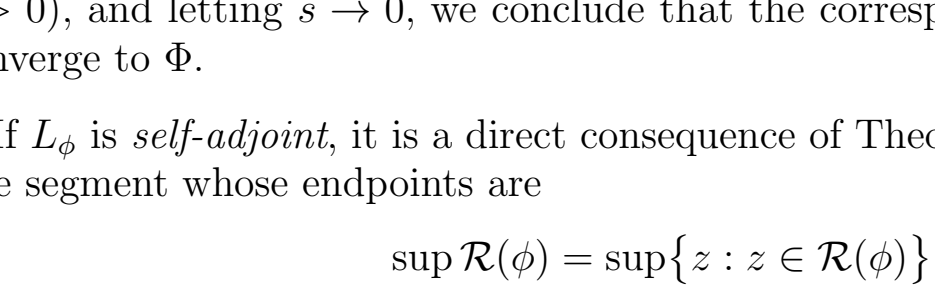

\title{
PRODUCTS OF LAURENT OPERATORS AND FIELDS OF VALUES
}

\author{
NATÁLIA BEBIANO $^{1^{*}}$ and JOÃO DA PROVIDÊNCIA ${ }^{2}$ \\ Communicated by I. M. Spitkovsky
}

\begin{abstract}
One of the most fundamental properties of the field of values of an operator is the inclusion of the spectrum within its closure. Obtaining information on the spectrum of products of operators in terms of this spectral inclusion region is a demanding issue. Stating general results seems difficult; however, conclusions can be derived in some special instances. In this paper, we show that the field of values of products of Laurent operators is easily related to the product of their fields of values, and the same occurs for certain classes of Laurent operators with matrix symbols. The results also apply to the class of infinite upper (lower) triangular Toeplitz matrices.
\end{abstract}

\section{INTRODUCTION}

Let $A$ be a bounded operator on a Hilbert space $H$ equipped with an inner product $\langle$,$\rangle . Denote by B(H)$ the algebra of bounded linear operators over $H$. In our discussion, we identify $H$ with $\mathbb{C}^{n}$ whenever $H$ has dimension $n$. The field of values of $A$ is the set of the complex plane defined as

$$
W(A)=\{\langle A f, f\rangle /\langle f, f\rangle: f \in H,\langle f, f\rangle \neq 0\} .
$$

This concept is a useful tool in studying linear operators, and it has been extensively investigated (see, e.g., [4] and the references therein).

Copyright 2016 by the Tusi Mathematical Research Group.

Received Mar. 4, 2016; Accepted Mar. 20, 2016.

${ }^{*}$ Corresponding author.

2010 Mathematics Subject Classification. Primary 46C20; Secondary 47A12, 47A10, 47B35.

Keywords. fields of values, spectrum, Toeplitz operator, Laurent operator, symbol. 


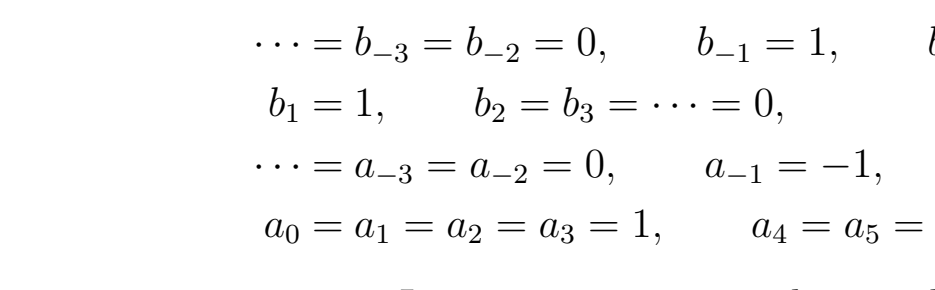

\title{
PRODUCTS OF LAURENT OPERATORS AND FIELDS OF VALUES
}

\author{
NATÁLIA BEBIANO $^{1^{*}}$ and JOÃO DA PROVIDÊNCIA ${ }^{2}$ \\ Communicated by I. M. Spitkovsky
}

\begin{abstract}
One of the most fundamental properties of the field of values of an operator is the inclusion of the spectrum within its closure. Obtaining information on the spectrum of products of operators in terms of this spectral inclusion region is a demanding issue. Stating general results seems difficult; however, conclusions can be derived in some special instances. In this paper, we show that the field of values of products of Laurent operators is easily related to the product of their fields of values, and the same occurs for certain classes of Laurent operators with matrix symbols. The results also apply to the class of infinite upper (lower) triangular Toeplitz matrices.
\end{abstract}

\section{INTRODUCTION}

Let $A$ be a bounded operator on a Hilbert space $H$ equipped with an inner product $\langle$,$\rangle . Denote by B(H)$ the algebra of bounded linear operators over $H$. In our discussion, we identify $H$ with $\mathbb{C}^{n}$ whenever $H$ has dimension $n$. The field of values of $A$ is the set of the complex plane defined as

$$
W(A)=\{\langle A f, f\rangle /\langle f, f\rangle: f \in H,\langle f, f\rangle \neq 0\} .
$$

This concept is a useful tool in studying linear operators, and it has been extensively investigated (see, e.g., [4] and the references therein).

Copyright 2016 by the Tusi Mathematical Research Group.

Received Mar. 4, 2016; Accepted Mar. 20, 2016.

${ }^{*}$ Corresponding author.

2010 Mathematics Subject Classification. Primary 46C20; Secondary 47A12, 47A10, 47B35.

Keywords. fields of values, spectrum, Toeplitz operator, Laurent operator, symbol. 


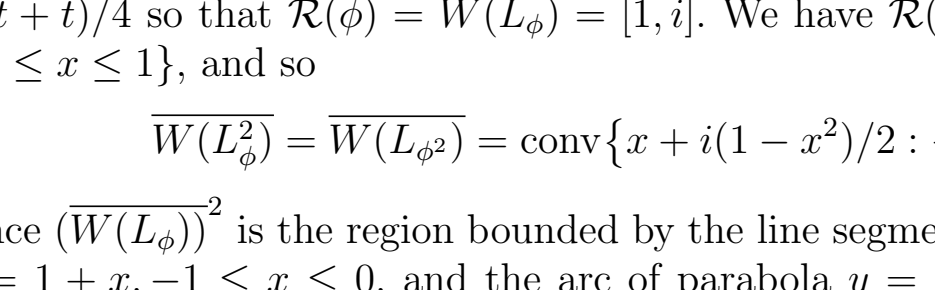

\title{
PRODUCTS OF LAURENT OPERATORS AND FIELDS OF VALUES
}

\author{
NATÁLIA BEBIANO $^{1^{*}}$ and JOÃO DA PROVIDÊNCIA ${ }^{2}$ \\ Communicated by I. M. Spitkovsky
}

\begin{abstract}
One of the most fundamental properties of the field of values of an operator is the inclusion of the spectrum within its closure. Obtaining information on the spectrum of products of operators in terms of this spectral inclusion region is a demanding issue. Stating general results seems difficult; however, conclusions can be derived in some special instances. In this paper, we show that the field of values of products of Laurent operators is easily related to the product of their fields of values, and the same occurs for certain classes of Laurent operators with matrix symbols. The results also apply to the class of infinite upper (lower) triangular Toeplitz matrices.
\end{abstract}

\section{INTRODUCTION}

Let $A$ be a bounded operator on a Hilbert space $H$ equipped with an inner product $\langle$,$\rangle . Denote by B(H)$ the algebra of bounded linear operators over $H$. In our discussion, we identify $H$ with $\mathbb{C}^{n}$ whenever $H$ has dimension $n$. The field of values of $A$ is the set of the complex plane defined as

$$
W(A)=\{\langle A f, f\rangle /\langle f, f\rangle: f \in H,\langle f, f\rangle \neq 0\} .
$$

This concept is a useful tool in studying linear operators, and it has been extensively investigated (see, e.g., [4] and the references therein).

Copyright 2016 by the Tusi Mathematical Research Group.

Received Mar. 4, 2016; Accepted Mar. 20, 2016.

${ }^{*}$ Corresponding author.

2010 Mathematics Subject Classification. Primary 46C20; Secondary 47A12, 47A10, 47B35.

Keywords. fields of values, spectrum, Toeplitz operator, Laurent operator, symbol. 


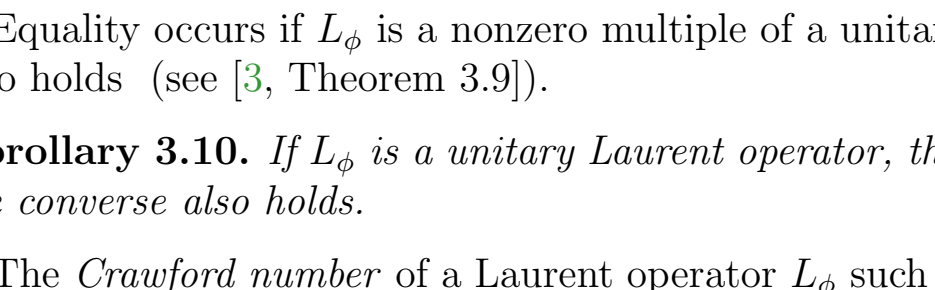

\title{
PRODUCTS OF LAURENT OPERATORS AND FIELDS OF VALUES
}

\author{
NATÁLIA BEBIANO $^{1^{*}}$ and JOÃO DA PROVIDÊNCIA ${ }^{2}$ \\ Communicated by I. M. Spitkovsky
}

\begin{abstract}
One of the most fundamental properties of the field of values of an operator is the inclusion of the spectrum within its closure. Obtaining information on the spectrum of products of operators in terms of this spectral inclusion region is a demanding issue. Stating general results seems difficult; however, conclusions can be derived in some special instances. In this paper, we show that the field of values of products of Laurent operators is easily related to the product of their fields of values, and the same occurs for certain classes of Laurent operators with matrix symbols. The results also apply to the class of infinite upper (lower) triangular Toeplitz matrices.
\end{abstract}

\section{INTRODUCTION}

Let $A$ be a bounded operator on a Hilbert space $H$ equipped with an inner product $\langle$,$\rangle . Denote by B(H)$ the algebra of bounded linear operators over $H$. In our discussion, we identify $H$ with $\mathbb{C}^{n}$ whenever $H$ has dimension $n$. The field of values of $A$ is the set of the complex plane defined as

$$
W(A)=\{\langle A f, f\rangle /\langle f, f\rangle: f \in H,\langle f, f\rangle \neq 0\} .
$$

This concept is a useful tool in studying linear operators, and it has been extensively investigated (see, e.g., [4] and the references therein).

Copyright 2016 by the Tusi Mathematical Research Group.

Received Mar. 4, 2016; Accepted Mar. 20, 2016.

${ }^{*}$ Corresponding author.

2010 Mathematics Subject Classification. Primary 46C20; Secondary 47A12, 47A10, 47B35.

Keywords. fields of values, spectrum, Toeplitz operator, Laurent operator, symbol. 


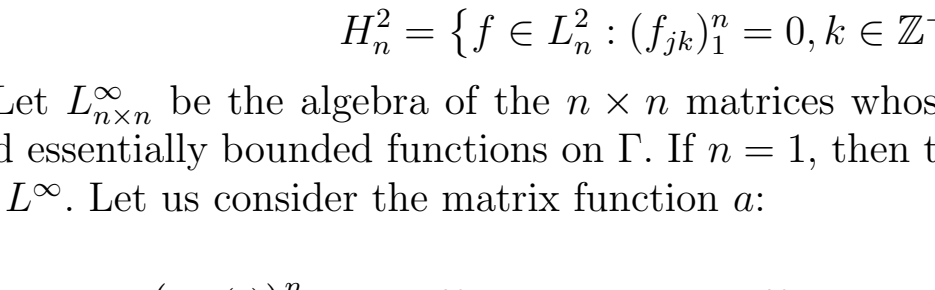

\title{
PRODUCTS OF LAURENT OPERATORS AND FIELDS OF VALUES
}

\author{
NATÁLIA BEBIANO $^{1^{*}}$ and JOÃO DA PROVIDÊNCIA ${ }^{2}$ \\ Communicated by I. M. Spitkovsky
}

\begin{abstract}
One of the most fundamental properties of the field of values of an operator is the inclusion of the spectrum within its closure. Obtaining information on the spectrum of products of operators in terms of this spectral inclusion region is a demanding issue. Stating general results seems difficult; however, conclusions can be derived in some special instances. In this paper, we show that the field of values of products of Laurent operators is easily related to the product of their fields of values, and the same occurs for certain classes of Laurent operators with matrix symbols. The results also apply to the class of infinite upper (lower) triangular Toeplitz matrices.
\end{abstract}

\section{INTRODUCTION}

Let $A$ be a bounded operator on a Hilbert space $H$ equipped with an inner product $\langle$,$\rangle . Denote by B(H)$ the algebra of bounded linear operators over $H$. In our discussion, we identify $H$ with $\mathbb{C}^{n}$ whenever $H$ has dimension $n$. The field of values of $A$ is the set of the complex plane defined as

$$
W(A)=\{\langle A f, f\rangle /\langle f, f\rangle: f \in H,\langle f, f\rangle \neq 0\} .
$$

This concept is a useful tool in studying linear operators, and it has been extensively investigated (see, e.g., [4] and the references therein).

Copyright 2016 by the Tusi Mathematical Research Group.

Received Mar. 4, 2016; Accepted Mar. 20, 2016.

${ }^{*}$ Corresponding author.

2010 Mathematics Subject Classification. Primary 46C20; Secondary 47A12, 47A10, 47B35.

Keywords. fields of values, spectrum, Toeplitz operator, Laurent operator, symbol. 


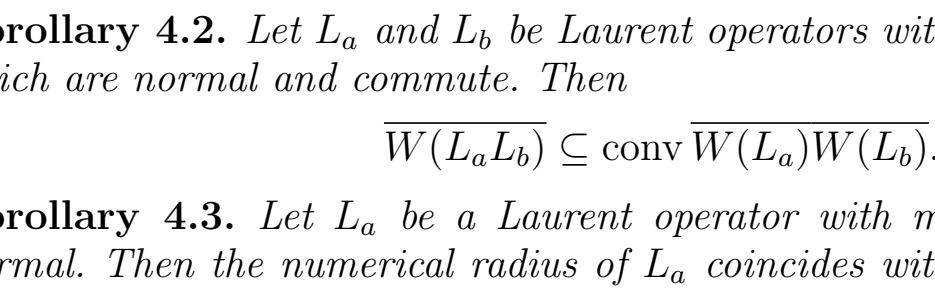

\title{
PRODUCTS OF LAURENT OPERATORS AND FIELDS OF VALUES
}

\author{
NATÁLIA BEBIANO $^{1^{*}}$ and JOÃO DA PROVIDÊNCIA ${ }^{2}$ \\ Communicated by I. M. Spitkovsky
}

\begin{abstract}
One of the most fundamental properties of the field of values of an operator is the inclusion of the spectrum within its closure. Obtaining information on the spectrum of products of operators in terms of this spectral inclusion region is a demanding issue. Stating general results seems difficult; however, conclusions can be derived in some special instances. In this paper, we show that the field of values of products of Laurent operators is easily related to the product of their fields of values, and the same occurs for certain classes of Laurent operators with matrix symbols. The results also apply to the class of infinite upper (lower) triangular Toeplitz matrices.
\end{abstract}

\section{INTRODUCTION}

Let $A$ be a bounded operator on a Hilbert space $H$ equipped with an inner product $\langle$,$\rangle . Denote by B(H)$ the algebra of bounded linear operators over $H$. In our discussion, we identify $H$ with $\mathbb{C}^{n}$ whenever $H$ has dimension $n$. The field of values of $A$ is the set of the complex plane defined as

$$
W(A)=\{\langle A f, f\rangle /\langle f, f\rangle: f \in H,\langle f, f\rangle \neq 0\} .
$$

This concept is a useful tool in studying linear operators, and it has been extensively investigated (see, e.g., [4] and the references therein).

Copyright 2016 by the Tusi Mathematical Research Group.

Received Mar. 4, 2016; Accepted Mar. 20, 2016.

${ }^{*}$ Corresponding author.

2010 Mathematics Subject Classification. Primary 46C20; Secondary 47A12, 47A10, 47B35.

Keywords. fields of values, spectrum, Toeplitz operator, Laurent operator, symbol. 